\title{
SPECTRAL CLASSIFICATION OF SOME LONG-PERIODAND SEMIREGULAR VARIABLES NEAR TIMES OF MAXIMUM
}

\author{
L. W. SIMON and W. BUSCOMBE \\ Department of Astronomy, Lindheimer Astronomical Research Center, Northwestern University, \\ Evanston, III., U.S.A.
}

(Read by P. Rybski)

\begin{abstract}
Spectra of 60 M-type long-period and semiregular variables, obtained near the time of maximum at Siding Spring Observatory in Australia, from 1965-1967, have been classified on the Keenan system.
\end{abstract}

Between September 1965 and November 1967, spectra of standard stars and longperiod and semiregular variables near their times of maximum were obtained on the Meinel Spectrograph at Siding Spring Observatory in Australia. They cover the wavelength region $3600-5100 \AA$ with a dispersion of $118 \AA \mathrm{mm}^{-1}$ on baked IIa-O emulsion. The spectra are widened $0.25 \mathrm{~mm}$. Sensitometer spots were exposed for each night of observation. The material includes 89 spectra of long-period and semiregular variables of types $\mathrm{K}$ and $\mathrm{M}$, and 26 (mostly irregular) of types $R, N$ and $S$.

The 60 variables listed below are classified on the system presented by Keenan (1966). Classifications for 41 of these stars are listed in Bidelman's catalogue of emission-line stars (1954). As seen in the diagram, there is a very good correlation between these classes and the Siding Spring classes. The radial velocities have been measured, and will be presented with further details of the spectra in Simon's doctoral dissertation.

TABLE I

Spectral classes of long-period and semiregular variables near times of maximum ${ }^{\mathrm{a}}$

\begin{tabular}{rllll} 
HD number & Name & $\begin{array}{l}\text { Spectral } \\
\text { class }\end{array}$ & $\begin{array}{l}\text { Type } \\
\text { b }\end{array}$ & $\begin{array}{l}\text { Julian date of } \\
\text { observation, } \\
2439000+\end{array}$ \\
\hline 151 & SW Scl & M2e & Sr & 452 \\
151 & SW Scl & M3 & Sr & 814 \\
409 & V Scl & M6e & M & 811 \\
1115 & S Scl & M6.5e & M & 808 \\
1760 & T Cet & M4 & SRb & 015 \\
1760 & T Cet & M4 & SRb & 017 \\
1760 & T Cet & M4 & SRb & 451 \\
1925 & S Tuc & M4e & M & 781 \\
5774 & U Tuc & M4e & M & 365 \\
6592 & Z Cet & M6e & M & 810 \\
6592 & Z Cet & M6.5e & M & 812 \\
17491 & Z Eri & M4e & SRb & 015 \\
17491 & Z Eri & M4 & SRb & 017 \\
17491 & Z Eri & M4 & SRb & 451 \\
17895 & RR Eri & M5 & SRb & 451 \\
& & & &
\end{tabular}


Table I (continued)

\begin{tabular}{|c|c|c|c|c|}
\hline HD number & Name & $\begin{array}{l}\text { Spectral } \\
\text { class }\end{array}$ & Type ${ }^{b}$ & $\begin{array}{l}\text { Julian date of } \\
\text { observation, } \\
2439000+\end{array}$ \\
\hline 18242 & R Hor & M7 & $\mathbf{M}$ & $365^{c}$ \\
\hline 18242 & R Hor & M8 & $\mathbf{M}$ & $808^{d}$ \\
\hline 18949 & T Hor & $\mathrm{M} 4 \mathrm{e}$ & $\mathrm{M}$ & 780 \\
\hline 18949 & T Hor & M4e & $\mathbf{M}$ & 814 \\
\hline 20646 & X Cet & M6.5 & $\mathrm{M}$ & 346 \\
\hline 24754 & T Eri & $\mathrm{M} 4 \mathrm{e}$ & $\mathbf{M}$ & 810 \\
\hline 25725 & V Eri & M6.5 & SRc & 163 \\
\hline 25725 & V Eri & M6.5 & SRc & 451 \\
\hline 29383 & R Ret & $\mathrm{M} 4 \mathrm{e}$ & $\mathbf{M}$ & 164 \\
\hline 29383 & R Ret & M5e & M & 780 \\
\hline 30551 & R Pic & M0e & SRa & 452 \\
\hline 33894 & S Pic & M6.5e & M & 165 \\
\hline 40913 & V 352 Ori & M6 & $\mathrm{Lb}$ & 165 \\
\hline 41698 & S Lep & M5 & $\mathrm{SRb}$ & 450 \\
\hline 41698 & S Lep & M4 & $\mathrm{SRb}$ & 808 \\
\hline 71793 & R Cha & $\mathrm{M} 4 \mathrm{e}$ & $\mathbf{M}$ & 636 \\
\hline 73766 & RV Hya & M4 & SRc & 165 \\
\hline 73766 & RV Hya & M4 & SRc & 223 \\
\hline 81137 & WY Vel & M3e P & & 165 \\
\hline 81137 & WY Vel & M3e P & & 225 \\
\hline 84474 & RR Hya & M42 & $\mathbf{M}$ & 224 \\
\hline$-21^{\circ} 2931$ & SU Hya & M4 & $\mathrm{SRb}$ & 223 \\
\hline 105266 & RW Vir & M5 & $\mathrm{Lb}$ & 165 \\
\hline 105266 & RW Vir & M4 & $\mathrm{Lb}$ & 224 \\
\hline 109372 & BO Mus & M4 & $\mathrm{Lb}$ & 165 \\
\hline 118767 & V 744 Cen & M5 & $\mathrm{Lb}$ & 227 \\
\hline 118767 & V 744 Cen & M5 & $\mathbf{L b}$ & 634 \\
\hline 120285 & W Hya & M7e & $\mathrm{SRa}$ & 224 \\
\hline 120460 & VX Cen & M4 & SR & 225 \\
\hline 121518 & V 412 Cen & M4 & $\mathrm{Lb}$ & 635 \\
\hline 138547 & RU Lib & MO & $\mathbf{M}$ & 633 \\
\hline 149234 & X Ara & M5 & M & 365 \\
\hline 329889 & RX Lup & $\mathrm{M} 4 \mathrm{e}$ & $\mathbf{M}$ & 226 \\
\hline 152476 & RS Sco & M6e & $\mathbf{M}$ & 636 \\
\hline 172301 & $\mathrm{U} \mathrm{CrA}$ & $\mathrm{M} 2 \mathrm{e}$ & $\mathbf{M}$ & 364 \\
\hline 192702 & RT Sgr & M6.5e & $\mathbf{M}$ & 364 \\
\hline 199003 & S Ind & M6e & $\mathbf{M}$ & 370 \\
\hline 199003 & $\mathrm{~S}$ Ind & M4e & $\mathbf{M}$ & 780 \\
\hline 201866 & W Ind & M4e & SRc & 786 \\
\hline 202306 & RR Aqr & M3e & $\mathbf{M}$ & 752 \\
\hline 207192 & R Gru & M6.5e & $\mathbf{M}$ & 364 \\
\hline 212537 & T Gru & M1e & $\mathbf{M}$ & 365 \\
\hline 212539 & S Gru & M5e & $\mathbf{M}$ & 780 \\
\hline 216907 & S Aqr & M6e & M & 810 \\
\hline 218541 & Y Scl & M6.5 & $\mathrm{SRb}$ & 814 \\
\hline 221433 & V Phe & M6.5e & $\mathbf{M}$ & 780 \\
\hline 224269 & R Phe & M4e & $\mathbf{M}$ & 752 \\
\hline 224269 & R Phe & M4e & $\mathrm{M}$ & 782 \\
\hline
\end{tabular}

a Including 9 observations of irregular variables

b General Catalogue of Variable Stars, 3rd edition

c Phase: - 106 days.

d Phase: - 72 days. 


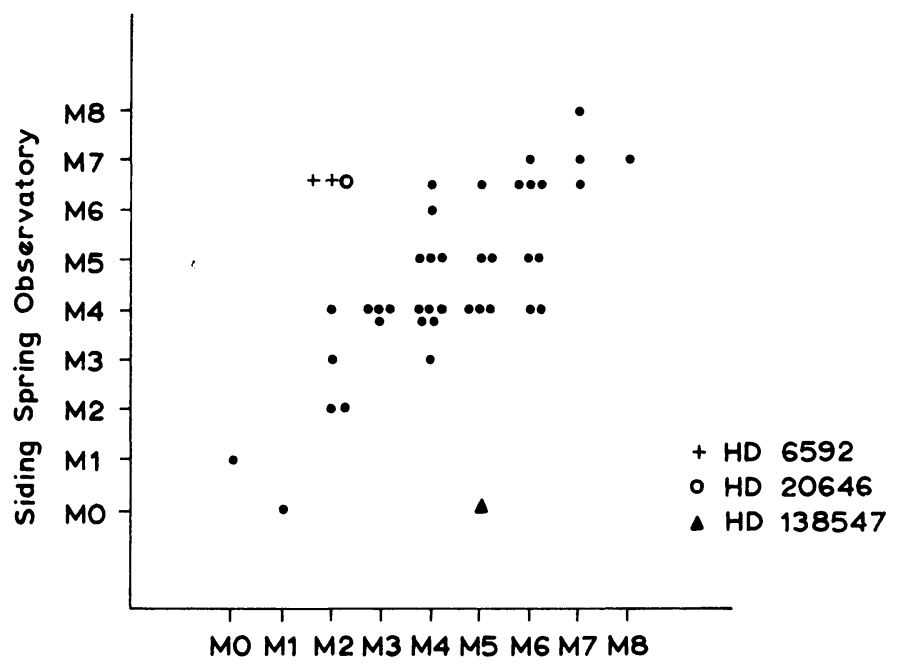

Fig. 1. Classification corre!ation.

\section{References}

Bidelman, W. P.: 1954, Astrophys. J. Suppl. Ser. 1, 175.

Keenan, P. C.: 1966, Astrophys. J. Suppl. Ser. 13, 333. 